\title{
GAZE BEHAVIOR DURING SIMULATED DRIVING: ELEMENTS FOR A VISUAL DRIVING AID
}

\author{
Daniel R. Mestre ${ }^{1}$, Franck Mars ${ }^{2}$, Sylvain Durand ${ }^{1}$, Fabrice Vienne $^{3}$, Stéphane Espié ${ }^{3}$ \\ ${ }^{1}$ UMR Mouvement et Perception \\ CNRS/ Université de la Méditerranée \\ Marseille, France \\ E-mail: mestre@1aps.univ-mrs.fr \\ ${ }^{2}$ Institut de Recherche en Communications et Cybernétique de Nantes (IRRCyN) \\ CNRS/Ecole Centrale de Nantes/Université de Nantes/Ecole Nationale Supérieure des \\ Techniques Industrielles et des Mines de Nantes \\ Nantes, France \\ ${ }^{3}$ Modélisations, Simulations et Simulateurs de Conduite \\ MSIS-CIR, INRETS \\ Arcueil, France
}

\begin{abstract}
Summary: Analyses of optic flow due to observer self-motion and analyses of the driver's gazing behavior during curve driving have suggested that the driver has a tendency to look at a location close to the tangent point on the inside edge of the road. Psychophysical experiments have further demonstrated that this visual strategy can be partly explained as an optimization of information pick-up. The main objective of the present study was to investigate, in an interactive simulation context, if this perceptual strategy might be used to define a visual aid for curve driving. In the framework of the French ARCOS project (Research action for secure driving; www.arcos2004.com), we used a mini-simulator developed by INRETS (MSIS-CIR group) in collaboration with FAROS company, with two main original characteristics: (1) during curve driving, the tangent point can be calculated and inserted in the visual scene in real-time and (2) a real-time eyerecording system (EYELINK, $\left.{ }^{\circledR} \mathrm{SMI}\right)$ allows us to evaluate the relationships between driving performance, gaze direction and the on-line presentation of the tangent point.
\end{abstract}

\section{INTRODUCTION}

The description of useful visual information for the control of self-motion remains a serious problem, notably in the field of car driving, due to the singularities and complexity of the road environment. In the 50's, Gibson (1958) introduced the concept of optic flow and suggested that our motion through the environment produces a pattern of optic flow that, in return, specifies the properties of our displacement.

In this general "feedback" (ex-proprioceptive) conception of visual information during driving, however, alternative sources of useful information for the control of self-motion have been suggested (see Wann \& Land, 2000; Fajen \& Warren, 2000, for an overview). Among potential visual cues for the control of self-motion during car driving, Gordon (1966) already noted that "...when the moving vehicle is aligned with the highway, each point on the road border and lane marker falls on the angular position previously occupied by another point of the border, and the road assumes a 'steady state appearance." Whereas drivers are keen to use optical flow to 
control their trajectory, they are equally susceptible to use optical stability to steer their vehicle, using edge lines. Driving corresponds in this case to a tracking task, the problem being to maintain visual stability of edge lines. Riemersma (1981) demonstrated that, during simulated road driving, edge line motion was an effective visual cue for the control of heading and lateral control. One interesting consequence of this is that, under nighttime conditions, delineation systems appear to be a privileged visual cue for facilitating driving on straight and curved roads. It is clear that, beside the global optic flow pattern, singularities in the road environment structure play a role in the perception and control of a car's trajectory.

Following this line of reasoning, Land and Lee (1994) introduced new methodological tools by recording gaze behavior during car driving. They demonstrated that, in curve driving, the eyes tend to fixate on the inside edge of the road near a point known as the "tangent" or "reversal" point of the road, which is a singular point where the inside of the curve changes direction (Figure 1). This suggests that subjects pick up useful information for the control of self-motion around this point. In recent psychophysical experiments (Mestre, 2001; Mestre \& Durand, 2001), we evaluated the ability of human observers to discriminate variations in their direction of selfmotion during simulated curvilinear trajectories, as a function of the part of the global optical flow field they were looking at. Results clearly show that discrimination thresholds are minimal when subjects look directly at the tangent point. As the horizontal angle of gaze departs from the tangent point, thresholds increase notably. These results confirm the idea that, in tasks such as curve driving, the tangent point of the curve acts as a singularity in the dynamic visual field, enabling optimal perception of the direction of self-motion.
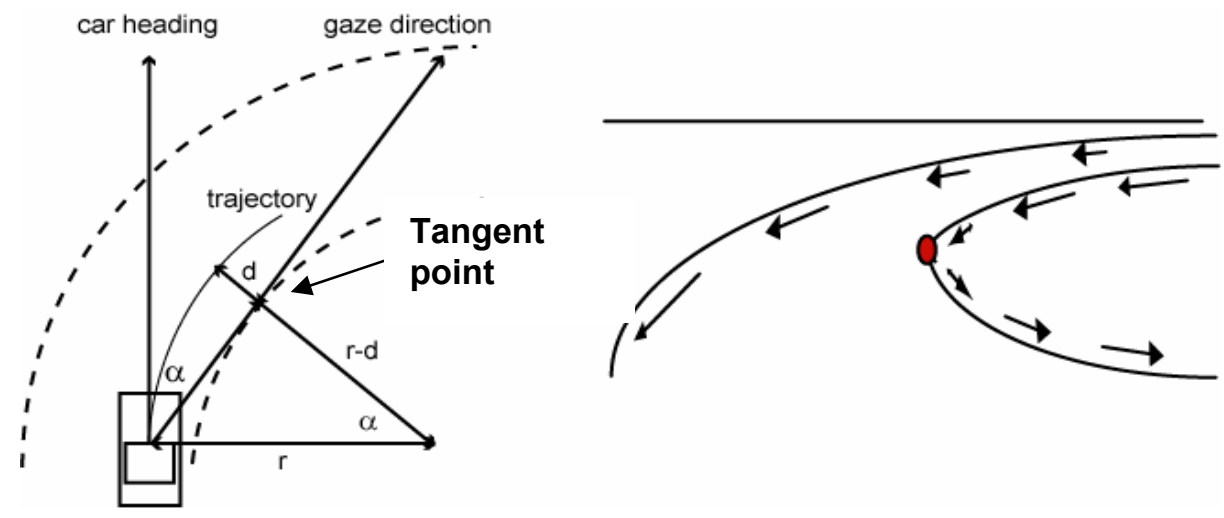

Figure 1. In a curve of radius $r$, the tangent point is the intersection point between the tangent to the inside edge line of the road (passing though the subject's point of view) and this edge line. On the right, a perspective view of the curve shows that the tangent point (red dot) is visually motionless when the observer's trajectory follows the road geometry (adapted from Land, 1998).

\section{EXPERIMENTAL STUDY}

In the present study, we aimed at searching whether the tangent could be used to define a visual aid for curve driving. In order to do so, we defined a simulation paradigm. We used an experimental simulation device, developed by INRETS (MSIS-CIR unit, Arcueil, France). 
During interactive simulations of car driving along a sinuous road, we investigated the effect of tangent point representation on driving performance while recording gaze behavior.

\section{Methods}

Subjects. Seven undergraduates, aged between 25 and 32 years, participated in the experiment. They all had normal vision (for correct eye recording). They all were active drivers for at least five years. Before the experiment, they signed an informed consent form.

Driving simulator. We used the driving simulator developed by INRETS in collaboration with the FAROS company (Figure 2, left). It enables full control of driving scenarios, real-time interactive driving, visual and auditory feedback, and on-line recording of simulated trajectories, for off-line analyses (Espié et al., 2003). The visualization part of the simulation software was upgraded, in order to allow real-time computation of the tangent point during curve driving, and superimposition of this tangent point in the visual environment during simulated driving (Figure 2 , right). The angular position of this point was derived from the driver's viewpoint with respect to the road geometry, and updated at each cycle of the simulation software.
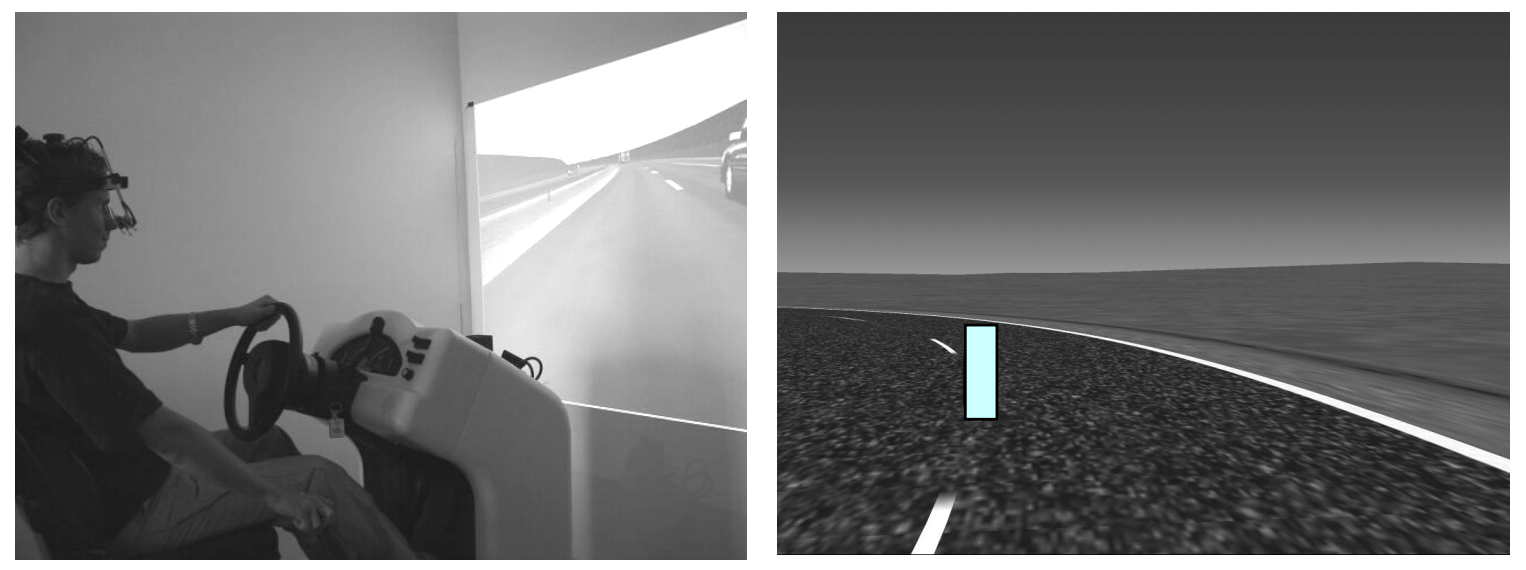

Figure 2. Left: Experimental set-up showing the simulator in front of a wide-angle visual scene representing the road environment. Right: Snapshot of the visual road environment. The tangent point (white vertical post) is situated on the dotted center line during a curve to the left. Its position in the visual scene is dependant upon the instantaneous driver's position and the road geometry.

Gaze recording. Gaze direction was measured on-line during the simulated driving task (Figure 2 , left) using a video-oculographic system "Eyelink" (Sensomotoric Instruments GmbH). Using this system, gaze direction is directly available, with reference to a pre-calibrated visual scene. The Eyelink system has the great advantage of allowing the subject to have his/her head free of motion while gaze is being monitored.

Experimental procedure. In a first step, subjects were trained on the simulator, during three trials on a road with radii of different curvatures, to the left and to the right, separated by portions of straight lines. This initial step insured that the subjects got used to this particular simulator and to sharp curve driving. They were instructed to drive at a sustained speed without leaving the right lane of the road (3.5 meters wide). Subjects were selected for the actual experimental sessions, 
provided they could drive the training road in "reasonable" time (as compared to a baseline defined by the experimenters) without running off the road.

The experiment itself consisted of three sessions, each lasting about 30 minutes. The road environment consisted of a randomly ordered succession of curves of various radii $(50,100,200$, 500 meters), with each radius appearing in each direction (right and left), resulting in 8 curves separated by portions of a straight line. The road edge lines were continuous, while the center line was discontinuous (Figure 2, right). In each session, three trials were conducted. In the first session, the tangent point was not represented. This enabled us to evaluate driving performance and gaze behavior in initial (no tangent point) conditions. In the second session, the tangent point was visible in the visual road environment (Figure 2, right). In the third session, it was no longer presented. This sequence of conditions aimed at separating potential effects (both on performance and gaze behavior) of the representation of the tangent point from strict learning effects. Gaze direction was always recorded.

\section{Data Analysis}

Data were analysed for each curve section of the experimental runway. Driving performance was quantified as the standard deviation of the lateral position of the simulated vehicle, with respect to the road centerline. This is a simple way to characterize the "stability" of a trajectory. Gaze behavior, during each curve, was measured as the average angular distance between gaze direction and angular position of the tangent point in the visual scene. Individual data were analyzed using repeated-measure analysis of the variance (ANOVA).

\section{RESULTS}

\section{Driving performance}

Subjects were required to stay in the right lane of the road. On average, the standard deviation (SD) of the lateral position (across sessions and subjects) is equal to $0.29( \pm 0.2)$ meters, meaning that the overall driving performance was quite satisfactory (the subjects managing to always stay in the right lane).

Data analysis reveals a main significant effect of curve direction (left versus right). Lateral position variability is equal to $0.32(+/-0.12)$ meters for curves to the left and to $0.27(+/-0.12)$ meters for curves to the right. This result suggests that left curves are harder to drive that right curves. It might be due to the fact that, on right curves, subjects visually rely on a continuous edge line, while on left curves, they are using a "dotted" line (Figure3), which might hamper the monitoring of its visual motion and/or the extraction of the tangent point visual motion.

However, it seems also plausible that drivers have a tendency to "cut" curves, and that requiring them to stay in the right lane in left curves might have induced lateral performance degradation. More specifically concerning the effect of tangent point visualization, there is a significant interaction effect between "curve direction" and "session" factors (Figure 3). 


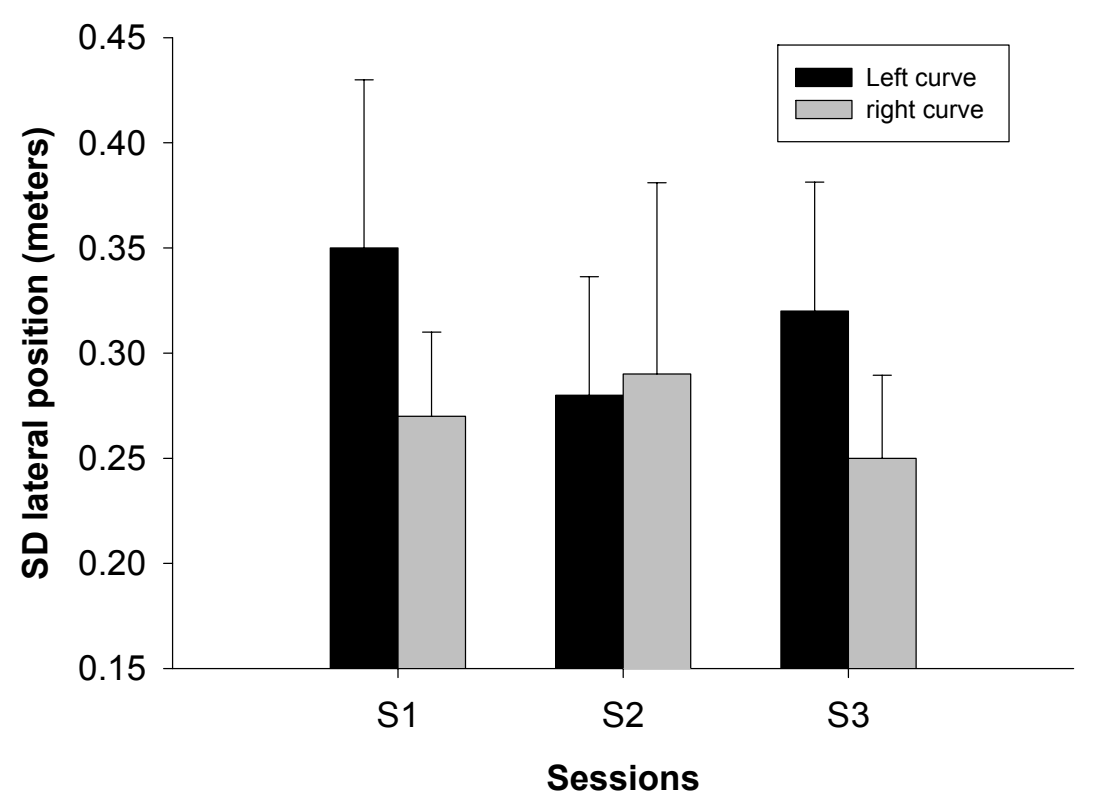

Figure 3. Average values of the SD of lateral position as a function of session, for left (black) and right (gray) curves

Figure 3 shows that lateral position variability is reduced when the tangent point is superimposed on the visual scene (session S2), in the case of left curves, for which initial performance is worse (S1), as compared to right curves. Such effect cannot be a simple "learning" effect, since lateral position variance for left curves increases in session 3, as compared to session 2, as the tangent point is no longer present (S3). No significant variation is observed for right curves. This might be related to a floor effect, right curve performance being "optimal" from session S1.

\section{Gaze behavior}

The main result here is that the average distance (across subjects and conditions) between gaze direction and tangent point is equal to about 4 degrees, and in all cases inferior to 8 degrees (see Figure 4). This simple result confirms Land \& Lee (1994) data, in showing that, in our simulation conditions, subjects also have a "natural" tendency to locate their gaze near the tangent point, during curve driving.

Figure 4 shows a decrease in the distance "gaze-tangent point" between Session S1 and Session S2 (when tangent point is visible), both for left and right curves. This index increases again between Session S2 and S3, as the tangent point is no longer present. This result is in line with what was observed concerning lateral position control. It further adds support to the suggestion that no learning affect may explain such a pattern of results. Gaze direction is close to the tangent point during curve driving, and this is more so when the tangent point is physically displayed in the visual road environment. 


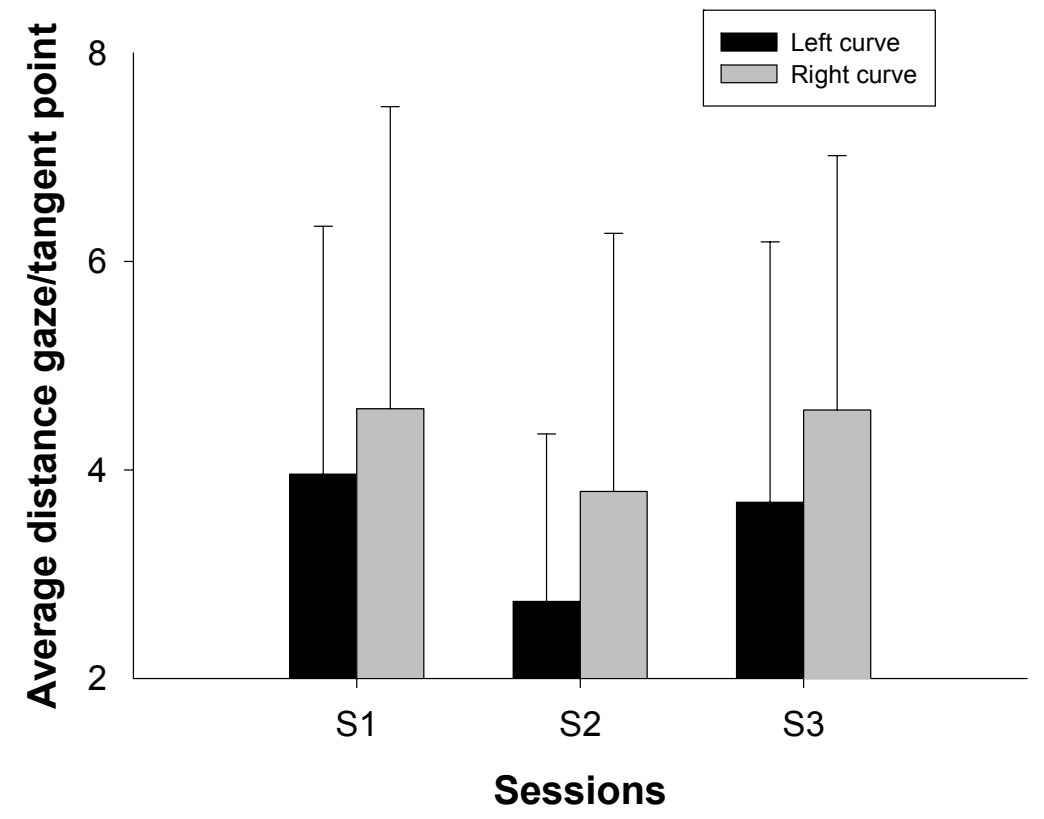

Figure 4. Average values of the average angular distance between gaze direction and tangent point, for the 3 sessions (S1 to S3), for left (black) and right (gray) curves

\section{CONCLUSIONS}

This paper further demonstrates the feasibility and interests of coupling eye movements recording systems to a driving simulator. Results confirm that, during curve driving, the tangent point to the inside of a curve "attracts" gaze and is useful information for the driver. They also suggest that its "on-line" figuration during curve driving might constitute an actual driving aid. This might be especially true for difficult situations, such as sharp curves in degraded visual conditions. This requires further investigations, including real-driving conditions, using head-up displays for instance.

Further analyses are also required to determine when and in what conditions it is beneficial to present the tangent point. It might be interesting to try to link the presentation of the tangent point to a deterioration of the trajectory. On a more fundamental level, a closer analysis of spatio-temporal aspects of the coupling between the direction of gaze and the location of the tangent point during a curve is also required. In this study, the analysis "unit" was the whole curve, and we presented only data concerning the average angular distance between gaze direction and the instantaneous location of the tangent point in the road scene. We already know from studies on car driving (i.e., Land \& Tatler, 2001), that anticipatory behaviors are involved during curve driving. For instance, gaze deviates toward the curve apex before the actual turn. In this study, we defined a curve from a geometrical point of view. A curve has to be defined from a more functional point of view, taking into account the driver's anticipatory motor and oculomotor behavior, both at the beginning and at the end of a curve. 


\section{ACKNOWLEDGMENTS}

This study was supported by PREDIT (Programme for Research, Experimentation and Innovation in land Transport), CNRS and the University of the Mediterranean.

\section{REFERENCES}

Espié, S., Mohellebi, H., Kheddar, A. (2003). A high performance / low-cost mini driving simulator alternative for human factor studies. Proceedings of HYPERLINK, "http://www.dsc-na.org/"DSC 2003 North America - Dearborn (USA), October 8-10, ISSN 1546-5071.

Fajen, B.R. \& Warren, W.H. (2000). Go with the flow. Trends in Cognitive Sciences, 10: 319324.

Gibson, J.J. (1958). Visually controlled locomotion and visual orientation in animals. British Journal of Psychology, 49: 182-194.

Gordon, D.A. (1966). Perceptual mechanisms in vehicular guidance. Public Roads, 34: 53-68.

Land, M. \& Lee, D.N. (1995). Which parts of the road guide steering? Nature, 377: 339-340.

Land, M. (1998). The visual control of steering. In, L.R. Harris \& M. Jenkin (Eds.), Vision and Action. Cambridge University Press, 163-180.

Land, M. \& Tatler, B. (2001). Steering with the head: The visual strategy of a racing driver. Current Biology, 11:1215-1220.

Mestre, D.R., Durand, S. (2001). Flow matters. Arvo Annual meeting, Fort Lauderdale, Florida, 29 April-4 May 2001. Investigative Ophthalmology and Visual Sciences, 42: 4977.

Mestre, D.R. (2001). Dynamic evaluation of the useful field of view in driving. Proceedings of the $1^{\text {st }}$ International Driving Symposium on Human Factors in Driver Assessment Training and Vehicle Design. Iowa City: University of Iowa, 234-239.

Riemersma, J.B.J. (1981). Visual control during straight road driving. Acta Psychologica, 48: 215-225.

Wann, J. \& Land, M. (2000). Steering with or without the flow. Is the retrieval of heading necessary? Trends in Cognitive Sciences, 8: 319-324. 